\title{
24-h Intraocular pressure control with evening-dosed travoprost/timolol, compared with latanoprost/timolol, fixed combinations in exfoliative glaucoma
}

\begin{abstract}
${ }^{1}$ Glaucoma Unit, Department of Ophthalmology, First University, Thessaloniki, Greece
\end{abstract}

${ }^{2}$ Department of Hygiene, Aristotle University of Thessaloniki, Thessaloniki, Greece

${ }^{3}$ Department of Ophthalmology, PRN Pharmaceutical Research Network, LLC, Charleston, SC, USA

Correspondence:

WC Stewart, Department of Ophthalmology, PRN Pharmaceutical Research Network, LLC, 6296 Rivers Avenue, Suite 309

Charleston, SC 29406, USA

Tel: + 843-762-6500;

Fax: + 843-762-7444

E-mail: info@prnorb.com

Received: 26 March 2010 Accepted in revised form: 7 June 2010

Published online: 23 July 2010

\begin{abstract}
Purpose To evaluate 24-h efficacy of travoprost/timolol fixed combination (TTFC) vs latanoprost/timolol fixed combination (LTFC) in exfoliative glaucoma (XFG). Design A prospective, single-masked, crossover, active-controlled, randomized 24-h comparison.

Methods After up to a 6-week medicine-free period, XFG patients were randomized to either TTFC or LTFC for 3 months, dosed each evening, and then changed to the opposite treatment for another 3 months. At the end of the washout, and both treatment periods, a 24-h intraocular pressure (IOP) curve was measured. Results In total, 40 patients completed the study. The TTFC group showed a lower mean absolute 24 -h IOP (18.7 \pm 2.6 vs $19.6 \pm 2.6 \mathrm{~mm} \mathrm{Hg}, P<0.001$ ), maximum IOP $(20.5 \pm 2.6$ vs $21.5 \pm 2.6 \mathrm{~mm} \mathrm{Hg}, P<0.001)$ and 24-h IOP range $(3.4 \pm 1.3$ vs $4.1 \pm 1.6 \mathrm{~mm} \mathrm{Hg}$, $P=0.01$ ). At individual time points, TTFC showed reduced IOPs compared with LTFC, after a Bonferroni correction, at 1000, 1800, and 2200 hours $(P \leqslant 0.04)$. No statistical differences existed at hours: 0600, 1400, and $0200(P \geqslant 0.05)$ and for the minimum IOP $(P=0.09)$.

Conclusions This study suggests that evening-dosed TTFC may provide greater 24-h IOP reduction, primarily at the $\mathbf{1 8 0 0}$ hours time point, compared with LTFC in XFG. Eye (2010) 24, 1606-1613; doi:10.1038/eye.2010.100; published online 23 July 2010
\end{abstract}

AGP Konstas'1, DG Mikropoulos', TA Embeslidis', AT Dimopoulos', A Papanastasiou', A-B Haidich² and WC Stewart ${ }^{3}$
Keywords: 24-h intraocular pressure; efficacy; travoprost/timolol fixed combination; latanoprost/timolol fixed combination; exfoliative glaucoma

Introduction

Recently, the travoprost/timolol maleate fixed combination (DuoTrav, Alcon Laboratories, Inc., Fort Worth, TX, USA) gained commercial approval in Europe. Barnebey et al $^{1}$ showed in a regulatory trial that morning-dosed travoprost/ timolol fixed combination (TTFC) provided a further reduction of intraocular pressure (IOP) of 0.9-2.4 mm Hg compared with evening-dosed travoprost.

In contrast, the latanoprost/timolol maleate fixed combination (Xalacom, Pfizer, Inc., New York City, NY, USA), the first prostaglandin/ timolol fixed combination available, showed a $1.1-1.2 \mathrm{~mm} \mathrm{Hg}$ difference between this preparation, dosed in the morning, to eveningdosed latanoprost. ${ }^{2,3}$ Therefore, it is possible that the travoprost-based fixed combination might have a slight efficacy advantage over the latanoprost-based product. Recent data by Topouzis et $a l^{4}$ showed little difference in a direct comparison between both these fixed combinations, when both were dosed in the morning, over a three-point diurnal curve.

Nonetheless, previous data by Konstas et $a l^{5-7}$ evaluating the latanoprost/timolol and TTFCs, have shown that evening dosing provides better 
24-h IOP control compared with the respective individual prostaglandin component. Therefore, evening dosing might better separate any potential efficacy differences between the latanoprost and travoprostbased fixed combinations.

The purpose of this study was to evaluate the 24-h IOP efficacy between evening-dosed TTFC and latanoprost/ timolol fixed combinations (LTFCs) in exfoliative glaucoma (XFG) patients.

\section{Materials and methods}

\section{Patients}

Patients were recruited for this prospective, singlemasked, crossover study from the Glaucoma Unit of the First University Department of Ophthalmology, AHEPA Hospital, Thessaloniki, Greece (Clinical Trial Number: NCT00757835). We included patients of either gender, older than 39 years of age, who had a clinical diagnosis of XFG in at least one eye (study eye) and, at baseline, an untreated IOP of $>25 \mathrm{~mm} \mathrm{Hg}$ at 1000 hours ( $\pm 1 \mathrm{~h}$ ). The clinical diagnosis of XFG relied on the clinical detection of exfoliation material visible on the anterior lens surface in a typical configuration and open, anterior chamber angles on indentation gonioscopy. ${ }^{8}$ All study patients had early-to-moderate XFG (for this study this was defined as glaucomatous disc damage with disc cupping not exceeding 0.8 and reproducible glaucomatous visual field loss $<14.0 \mathrm{~dB}$ in the study eye with Humphrey 24-2 automated perimetry).

We excluded XFG patients who received therapy if they had a previous history of unresponsiveness (deemed to be a morning IOP reduction of $<10 \%$ ) to any anti-glaucoma medication, including LTFC or timolol, or there was a history of non-adherence. Patients were also excluded if they showed: unreliable applanation tonometry; inadequate visualization of the ocular fundus or anterior chamber; a concurrent infectious / noninfectious conjunctivitis, keratitis, or uveitis; a history of hypersensitivity to any components of the preparations used in this trial; woman of child-bearing potential or not using reliable birth control; pregnancy or lactation; a clinically severe medical or psychiatric condition; participation (or current participation) in any investigational drug or device trial within the previous 3 months before the screening visit; an intraocular conventional surgery or laser surgery in the participating eye; risk of visual field or visual acuity worsening as a consequence of participation in the trial; an inability to give informed consent; anticipated change in systemic hypotensive therapy during the active treatment portion of the trial; progressive retinal or optic nerve disease apart from glaucoma; unwillingness to accept the risk of iris colour or eyelash changes; risk for uveitis or cystoid macular oedema in this trial; or history of ocular herpes simplex, reactive airway disease, second- or third-degree heart block, poorly compensated congestive heart failure or concomitant use of systemic $\beta$-blockers.

\section{Methods}

All patients signed an informed consent agreement approved by an institutional review board (Bioethics Committee of the Medical School, Aristotle University of Thessaloniki, Thessaloniki, Greece) before any procedures were performed. The Treaty of Helsinki was followed for this study. At visit 1, subjects had an ophthalmic and systemic history taken and had dilated funduscopy, gonioscopy, and automated full threshold perimetry performed (Humphrey 24-2 test, SITA standard). At this visit, as well as at all other visits, the IOP (two measurements at 1000 hours $\pm 1 \mathrm{~h}$ were averaged) was measured and Snellen visual acuity and slit lamp biomicroscopy were performed. This time was chosen as the peak pressure in Greece based on previous data. ${ }^{9,10}$ Qualified XFG patients were then washed out of their glaucoma medications in both eyes and asked to return in 6 weeks for patients on previous prostaglandin therapy, and 4 weeks for those on previous $\beta$-blocker therapy, for the baseline visit (visit 2).

At visit 2, and at all other 24-h monitoring visits (visits 4 and 6), patients had sitting IOP measurements at 0600 , $1000,1400,1800,2200$, and 0200 hours. Patients who met the IOP entry requirements were randomly assigned, by a computer generated randomized number list, to receive either the TTFC or LTFC for the first 3-month treatment period ( \pm 2 weeks) to be instilled one drop to the study eye at 2000 hours. No washout period was used between groups. The study was designed to accomplish the washout of the period 1 medicine over the 3-month treatment of period 2. ${ }^{11}$

A safety evaluation was performed after 2 weeks of treatment (visit 3). At the end of period 1, a 24-h curve was again performed (visit 4). Patients were then switched to the second study medicine for period 2 . A safety visit was again performed after 2 weeks of treatment (visit 5) and a final 24-h curve was performed at the end of the second 3-month ( \pm 2 weeks) treatment period (visit 6).

The same investigators at the clinical site measured the IOP and used the same calibrated instrument (Goldmann Applanation Tonometer, Haag-Streit, Koeniz, Switzerland) to perform the 24-h monitoring of the IOP. During the study, the investigators, staff, and patients were masked to the treatment regimen. A dosing coordinator allocated the medications to the patients 
according to a code and did not participate in this trial in any other way. This was a single-masked study. The commercially available medicine bottles were masked by placing a label over the medicine bottle. Patients were instructed not to show the labeled medicine bottle to the staff or investigators. The medicine was single masked because of the cost in time and in money to arrange for sterile transfer of both treatments to similar unmarked bottles. The staff was masked to the study medicine except a dosing coordinator.

Patients were admitted to the hospital in the morning and measurements were recorded at 1000, 1400, 1800, 2200, 0200, and 0600 hours. At the 2200 hours

measurement, patients were awake at bed rest. The 0200 and 0600 hours IOP measurements were performed immediately after wakening and at the slit lamp. Patients were encouraged to carry out a normal life as much as possible within the hospital boundaries.

Patients were instructed regarding correct medication instillation and adherence. In this study, all patients were instructed to perform nasolacrimal occlusion for $1 \mathrm{~min}$ after instillation of each study eye drop. At each visit, local and systemic side effects that occurred during the treatment period were recorded. Side effects were evaluated by asking patients a general query regarding their state of health. Patients also were asked their compliance to the study medicine using a general query.

\section{Statistics}

Statistical analyses compared the primary efficacy variable, mean 24-h IOP curve (the average pressure for the six time points evaluated), using a repeated measures of analysis. Individual time points were evaluated with a paired $t$-test within the analysis of variance (ANOVA), in case of significant treatment and time effect. ${ }^{12}$ A modified Bonferroni-correction was used to adjust $P$-values for multiple comparisons in all individual time points using a range of corrections from $\alpha / 1-6$ depending on the respective $P$-value rank. ${ }^{13}$ The significance level was set at $5 \%$ and a two-way analysis was used for all other tests.

As calculated from the PS programme, this 24-h study had an $80 \%$ power to identify a $1.25 \mathrm{~mm} \mathrm{Hg}$ difference between individual time points and between mean 24-h pressures assuming a SD of $2.8 \mathrm{~mm} \mathrm{Hg}$ between treatments. ${ }^{14-16}$ In those patients that both eyes qualified, only one eye was randomly selected for analysis.

The secondary primary efficacy variables for pressure, the mean 24-h range (average of the highest time point minus the lowest time point for each individual patient) as well as the mean maximum and minimum pressures were analyzed by a paired $t$-test. In addition, baseline characteristics were compared in patients first started on
TTFC than on latanoprost/timolol maleate fixed combination using unpaired $t$-test for quantitative variables and $\chi^{2}$ test or Fisher's exact test, when the expected cell count was $<5$, for qualitative variables. A correlation coefficient was used to analyze the reduction in the IOP. ${ }^{12}$ Adverse events were evaluated by a McNemar's test, a paired $\chi^{2}$ test, because of the crossover design of the study. ${ }^{17}$ Analyses were conducted using SPSS 15.0 (SPSS, Inc., Chicago, IL, USA).

\section{Results \\ Patients}

Patient characteristics of those included in this study are shown in Table 1. In all, 40 phakic patients completed the study of 44 enrolled. Four patients were discontinued from study medicine before the completion of both 24-h IOP curves; for either adverse events (two in the travoprost/timolol period, one in the latanoprost/ timolol period), and one patient was lost to follow-up. In total, 22 were randomized to TTFC and 18 to LTFC treatment in period 1 . The baseline characteristics of the treatment groups were similar except for the age $(P=0.002)$ and the visual acuity $(P=0.04)$.

\section{Intraocular pressure}

The mean IOP and the pressure reductions from baseline are shown in Tables 2 and 3, as well as Figure 1. The IOP was significantly reduced from untreated baseline at each individual time point and for the mean 24-h pressure for both treatment groups $(P<0.001)$. When treatments were compared directly, for the primary efficacy variable, the $24-h$ average pressure, the TTFC showed a lower absolute IOP levels for the 24-h curve $(P<0.001$, Table 2$)$. The difference between the two medicines was $0.9 \pm 1.2 \mathrm{~mm} \mathrm{Hg}$. This was true also for the maximum pressure $(20.5 \pm 2.6$ vs $21.5 \pm 2.6 \mathrm{~mm} \mathrm{Hg}$, $P<0.001)$ and for the 24 -h range of pressure $(3.4 \pm 1.3$ vs $4.1 \pm 1.6 \mathrm{~mm} \mathrm{Hg}, P=0.01$ ).

There was a significant treatment effect $(P<0.001)$ and time effect $(P=0.01)$ within the ANOVA, therefore the treatments were compared at individual time points in these post hoc analyses. There was also a significant period effect $(P=0.02)$ with both medicines showing a slightly lower pressure at the end of period 2 compared with period 1 . However, there was no carry over effect so the treatment order did not affect the IOP results $(P=0.74)$.

At individual time points, the TTFC reduced the IOP better than the LTFC, after a modified Bonferroni correction, at 1000, 1800, and 2200 hours $(P \leqslant 0.04)$. In contrast, no statistical differences existed at hours: 0600 , 1400 , and $0200(P \geqslant 0.05)$ and for the minimum pressure 
Table 1 Overall baseline characteristics and comparison between treatments

\begin{tabular}{|c|c|c|c|c|}
\hline Characteristic & Baseline $\mathrm{N}=40$ & TTFC $\mathrm{N}=22^{\mathrm{a}}$ & $L T F C N=18^{\mathrm{a}}$ & P-value \\
\hline Male & $19(47)$ & $11(50)$ & $10(56)$ & 0.76 \\
\hline Female & $21(53)$ & $11(50)$ & $8(44)$ & \\
\hline Mean age (years) & $68.2 \pm 8.4$ & $64.7 \pm 8.8$ & $72.6 \pm 6.0$ & 0.002 \\
\hline Mean morning baseline intraocular pressure $(\mathrm{mm} \mathrm{Hg})$ & $31.3 \pm 2.8$ & $29.9 \pm 2.4$ & $30.3 \pm 3.3$ & 0.64 \\
\hline Mean Snellen best-corrected visual acuity & $0.8 \pm 0.2$ & $0.9 \pm 0.2$ & $0.7 \pm 0.3$ & 0.04 \\
\hline Mean vertical cup/disc ratio & $0.6 \pm 0.1$ & $0.6 \pm 0.1$ & $0.6 \pm 0.1$ & 0.80 \\
\hline Mean visual field loss mean deviation $(\mathrm{dB})$ & $-7.5 \pm 3.3$ & $-7.7 \pm 3.4$ & $-7.4 \pm 4.4$ & 0.85 \\
\hline Mean corneal thickness & $539 \pm 27$ & $534 \pm 26$ & $545 \pm 27$ & 0.22 \\
\hline Most common previous therapies & & & & 0.44 \\
\hline Dorzolamide/timolol fixed combination & $11(27)$ & $6(27.3)$ & $5(27.8)$ & \\
\hline TTFC & $5(12)$ & $4(18.2)$ & $1(5.6)$ & \\
\hline Bimatoprost/timolol fixed combination & $4(10)$ & $2(9.1)$ & $2(11.1)$ & \\
\hline Travoprost & $4(10)$ & $3(13.6)$ & $1(5.6)$ & \\
\hline LTFC & $3(7)$ & $0(0)$ & $3(16.7)$ & \\
\hline Dorzolamide/timolol fixed combination + travoprost & $3(7)$ & $1(4.5)$ & $2(11.1)$ & \\
\hline Other & $10(25)$ & $6(27.3)$ & $4(22.2)$ & \\
\hline
\end{tabular}

Abbreviations: LTFC, latanoprost/timolol fixed combination; TTFC, travoprost/timolol fixed combination.

Data are shown as mean \pm SD, or as number of patients $(\%)$.

aThese two columns represent first period treatment characteristics before changing to the opposite treatment.

Table 2 Absolute intraocular pressure levels ( $P$-values are adjusted for the Bonferroni correction so any value $<0.05$ is statistically significant)

\begin{tabular}{lccccc}
\hline & Baseline & TTFC & LTFC & Mean difference $^{\text {P-value }}$ \\
\hline Mean 24-h & $28.5 \pm 2.6$ & $18.7 \pm 2.6$ & $19.6 \pm 2.6$ & $-0.9 \pm 1.2$ & $<0.001$ \\
0600 hours & $29.7 \pm 3.5$ & $19.1 \pm 2.6$ & $19.7 \pm 2.3$ & $-0.6 \pm 1.5$ & 0.06 \\
1000 hours & $30.4 \pm 3.5$ & $18.3 \pm 2.3$ & $19.2 \pm 2.3$ & $-0.8 \pm 1.8$ & 0.04 \\
1400 hours & $28.7 \pm 2.9$ & $18.7 \pm 2.6$ & $19.6 \pm 2.9$ & $-0.9 \pm 2.4$ & 0.05 \\
1800 hours & $28.7 \pm 3.2$ & $18.6 \pm 2.6$ & $20.3 \pm 2.9$ & $-1.7 \pm 2.6$ & $<0.001^{\mathrm{b}}$ \\
2200 hours & $27.1 \pm 2.9$ & $18.6 \pm 3.2$ & $19.3 \pm 3.2$ & $-0.7 \pm 1.8$ & 0.04 \\
0200 hours & $26.6 \pm 4.5$ & $18.8 \pm 3.2$ & $19.4 \pm 3.9$ & $-1.1 \pm 1.5$ & 0.05 \\
Maximum & $31.9 \pm 3.2$ & $20.5 \pm 2.6$ & $21.5 \pm 2.6$ & $-0.4 \pm 1.6$ & $<0.001$ \\
Minimum & $25.3 \pm 2.6$ & $17.0 \pm 2.3$ & $17.5 \pm 2.6$ & $-0.6 \pm 1.5$ & 0.09 \\
24-h range & $6.6 \pm 2.6$ & $3.4 \pm 1.3$ & $4.1 \pm 1.6$ & & 0.01 \\
\hline
\end{tabular}

Abbreviations: LTFC, latanoprost/timolol fixed combination; TTFC, travoprost/timolol fixed combination.

abetween treatments.

bonferroni adjusted $P$-values.

Data are shown as mean \pm SD $(\mathrm{mm} \mathrm{Hg})$.

Table 3 Intraocular pressure reduction from baseline

\begin{tabular}{lrrcc}
\hline & \multicolumn{1}{c}{ TTFC } & \multicolumn{1}{c}{ LTFC } & Mean difference & P-value $^{\mathrm{a}}$ \\
\hline Mean 24-h & $9.8 \pm 2.3$ & $8.9 \pm 1.9$ & $-0.9 \pm 1.2$ & $<0.001$ \\
0600 hours & $10.6 \pm 2.6$ & $10.0 \pm 2.9$ & $-0.6 \pm 1.5$ & 0.06 \\
1000 hours & $12.1 \pm 3.5$ & $11.2 \pm 3.2$ & $-0.8 \pm 1.8$ & 0.04 \\
1400 hours & $10.1 \pm 3.2$ & $9.1 \pm 2.6$ & $-0.9 \pm 2.4$ & 0.05 \\
1800 hours & $10.0 \pm 3.2$ & $8.3 \pm 2.9$ & $-1.7 \pm 2.6$ & $<0.001^{\mathrm{b}}$ \\
2200 hours & $8.6 \pm 3.2$ & $7.9 \pm 3.2$ & $-0.7 \pm 1.8$ & 0.04 \\
0200 hours & $7.8 \pm 3.5$ & $7.2 \pm 3.5$ & $0.6 \pm 1.8$ & 0.05 \\
\hline
\end{tabular}

Abbreviations:LTFC, latanoprost/timolol fixed combination; TTFC, travoprost/timolol fixed combination.

aBetween treatments.

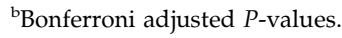

Data are shown as mean $\pm \mathrm{SD}(\mathrm{mm} \mathrm{Hg})$.

$(P=0.09)$. When the pressure reduction from untreated baseline was compared between treatment groups

(Table 3), the findings mirrored those of the absolute IOP.
Further, there was no correlation of IOP to corneal thickness for TTFC $(r=0.17, P=0.31)$ or LTFC treatment group ( $r=0.23, P=0.15)$. The baseline and mean corneal thickness measurements are shown in Table 1.

\section{Adverse events}

There was no statistical difference between the two treatment groups for any adverse event (Table 4). The most common adverse event detected was conjunctival hyperaemia, which was found in $10 \%(n=4)$ of patients treated with LTFC compared with $20 \%(n=8)$ when patients were treated with the TTFC $(P=0.29)$. In all, 22 adverse events were detected in the LTFC group and 27 were noted in the TTFC therapy. There were no serious adverse events recorded in this study. 


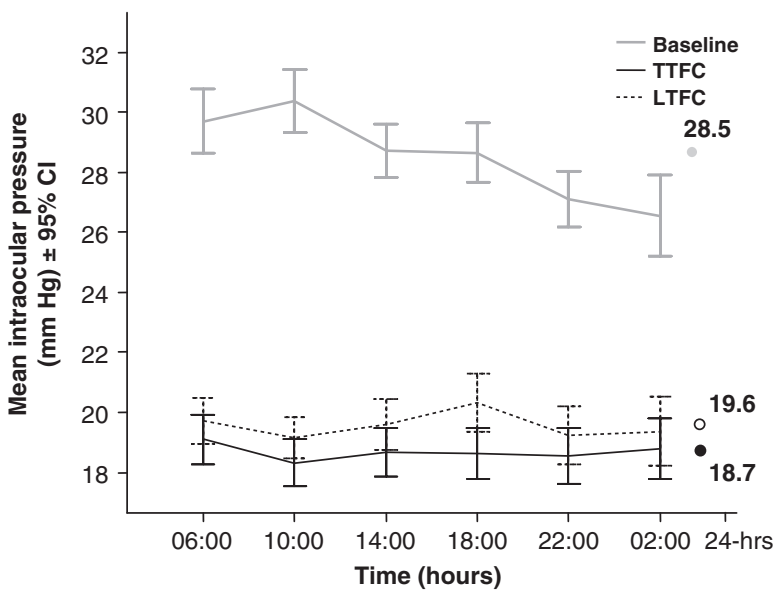

Figure 1 The figure shows the mean IOP $\pm 95 \%$ confidence interval at each individual time point and for the $24-\mathrm{h}$ pressure for untreated baseline (grey line), travoprost/timolol fixed combination (TTFC, solid black line), and latanoprost/timolol fixed combination (LTFC, dotted line) treatment groups.

\section{Discussion}

XFG is a common, sight-threatening form of glaucoma, which develops as a consequence of an age-related malfunction of the extracellular matrix. ${ }^{18}$ The worldwide number of individuals who suffer with XFG or exfoliation syndrome is between 60 and 70 million. ${ }^{18} \mathrm{XFG}$ is believed to be a more severe form of glaucoma, with a worse long-term prognosis, than primary open-angle glaucoma. This is thought to be due to the worse 24-h pressure characteristics observed in XFG. ${ }^{19-21}$

However, the higher level of pressure associated with XFG may have the advantage of allowing for a greater separation between therapies when performing clinical trials. In a separate $24-\mathrm{h}$ study, a significantly better mean 24-h pressure with travoprost $(0.6 \mathrm{~mm} \mathrm{Hg})$ vs latanoprost was noted in XFG primarily because of improved efficacy in the late afternoon and evening with travoprost. ${ }^{22}$ However, a separate trial by Parmaksiz et $a l^{23}$ did not find a difference in pressure reduction in XFG patients in a three-point diurnal curve. Nonetheless, published evidence suggests that because of the higher 24-h pressure curve characteristics, evaluating patients with XFG might help elucidate if a difference exists in ocular hypotensive efficacy between two glaucoma preparations.

The purpose of this study was to evaluate the 24-h IOP efficacy between evening-dosed TTFC and LTFC specifically in XFG patients.

This crossover study showed that both the TTFCs and the LTFCs reduced the IOP from untreated baseline over the 24-h pressure curve and for each individual time points. However, when these fixed combinations were compared directly, the travoprost-based fixed combination provided a lower pressure over the $24 \mathrm{~h}$, a lower maximum pressure and a lower range of the pressure. In addition, following a Bonferroni correction, individual time points at 1000, 1800, and 2000 hours were statistically lower with the TTFC. In contrast, no statistically significant difference was observed between groups at the other time points and for the minimum pressure.

These findings differ from those of Topouzis et al ${ }^{4}$ who found, on the final efficacy visit, no difference between the latanoprost and travoprost-based fixed combinations except for the pooled 0900 hours time point when TTFC was better. However, the study design between the earlier trial and our study differed in several ways. The crossover design of the current trial allowed us to compare the 24-h IOP characteristics of the two fixed combinations in the same eyes. Further, our study carried out a complete 24-h pressure evaluation, which may allow better separation between two medications.

In addition, we dosed the two fixed combinations in the evening. Previous studies have shown that when the prostaglandin-based fixed combinations were dosed in the evening a greater separation could be observed from its individual components, or compared with morning dosing. ${ }^{5,6,24,25}$ These 24 -h studies have suggested that prostaglandin analogues show a peak effect 12 to $24-\mathrm{h}$ after dosing. ${ }^{24-26}$ Consequently, when dosed in the evening the peak effect of the medicine may occur in the following daytime hours when pressures tend at the highest by most studies. A retrospective, cross-sectional study found TTFC to provide significantly lower pressure than latanoprost/timolol maleate fixed combination 24-h after instillation. ${ }^{27}$

Finally, our study population consisted of XFG patients vs primary open-angle glaucoma and ocular hypertension patients in the Topouzis trial. ${ }^{4}$ As stated previously, previous research has shown that XFG patients typically show higher pressures than those with primary open-angle glaucoma. ${ }^{9,10}$ This may have allowed for a greater reduction in pressure and a greater potential to differentiate any differences between these two fixed combination preparations. Other factors potentially related to the greater efficacy of the TTFC in our study remain unknown and highly speculative. Travoprost is known to bind to the receptor more strongly that does latanoprost. ${ }^{28}$ This might allow for more prolonged contact with the prostanoid receptor and might have provided the greater efficacy especially at the end of the dosing cycle at 1800 hours as observed in this trial and in a previous study by Konstas et al. ${ }^{22}$

What does the approximately $1 \mathrm{~mm} \mathrm{Hg}$ diurnal mean clinically? Since short-term IOPs are an excepted marker for long-term clinical outcomes in glaucoma one has to 
Table 4 Adverse events recorded in the study

\begin{tabular}{|c|c|c|c|c|c|}
\hline \multirow[t]{2}{*}{ Adverse eventr ${ }^{\mathrm{a}}$} & \multirow[t]{2}{*}{ LTFC } & \multicolumn{2}{|c|}{ TTFC } & \multirow[b]{2}{*}{ Total } & \multirow[t]{2}{*}{ P-value } \\
\hline & & Yes & No & & \\
\hline \multirow[t]{3}{*}{ Conjunctival hyperemia } & Yes & 2 & 2 & $4(10)$ & \multirow[t]{3}{*}{0.29} \\
\hline & No & 6 & 30 & $36(90)$ & \\
\hline & Total & $8(20)$ & $32(80)$ & $40(100)$ & \\
\hline \multirow[t]{3}{*}{ Stinging } & Yes & 3 & 3 & $6(15)$ & \multirow[t]{3}{*}{0.62} \\
\hline & No & 1 & 33 & $34(85)$ & \\
\hline & Total & $4(10)$ & $36(90)$ & $40(100)$ & \\
\hline \multirow[t]{3}{*}{ Hypertrichosis } & Yes & 3 & 0 & $3(7)$ & \multirow[t]{3}{*}{0.25} \\
\hline & No & 3 & 34 & $37(93)$ & \\
\hline & Total & $6(15)$ & $34(85)$ & $40(100)$ & \\
\hline \multirow[t]{3}{*}{ Skin pigmentation } & Yes & 0 & 0 & $0(0)$ & \multirow[t]{3}{*}{0.25} \\
\hline & No & 3 & 37 & $40(100)$ & \\
\hline & Total & $3(7)$ & $37(93)$ & $40(100)$ & \\
\hline \multirow[t]{3}{*}{ Itchiness } & Yes & 0 & 3 & $3(7)$ & \multirow[t]{3}{*}{0.25} \\
\hline & No & 0 & 37 & $37(93)$ & \\
\hline & Total & $0(0)$ & $40(100)$ & $40(100)$ & \\
\hline \multirow[t]{3}{*}{ Watering } & Yes & 1 & 0 & $1(2)$ & \multirow[t]{3}{*}{1} \\
\hline & No & 1 & 38 & $39(98)$ & \\
\hline & Total & $2(5)$ & $38(95)$ & $40(100)$ & \\
\hline \multirow[t]{3}{*}{ Superficial punctuate keratitis } & Yes & 1 & 1 & $2(5)$ & \multirow[t]{3}{*}{1} \\
\hline & No & 0 & 38 & $38(95)$ & \\
\hline & Total & $1(2)$ & $39(98)$ & $40(100)$ & \\
\hline \multirow[t]{3}{*}{ Dry eye sensation } & Yes & 0 & 1 & $1(2)$ & \multirow[t]{3}{*}{1} \\
\hline & No & 2 & 37 & $39(98)$ & \\
\hline & Total & $2(5)$ & $38(95)$ & $40(100)$ & \\
\hline \multirow[t]{3}{*}{ Eyelid swelling } & Yes & 1 & 1 & $2(5)$ & \multirow[t]{3}{*}{1} \\
\hline & No & 0 & 38 & $38(95)$ & \\
\hline & Total & $1(2)$ & $39(98)$ & $40(100)$ & \\
\hline
\end{tabular}

Abbreviations: LTFC, latanoprost/timolol fixed combination; TTFC, travoprost/timolol fixed combination.

asome patients experienced multiple adverse events.

Data are shown as number of patients (\%).

project long-term results. Several studies have noted that $1 \mathrm{~mm} \mathrm{Hg}$ differences over 5 years can alter the incidence of progression in primary open-angle and exfoliation patients. ${ }^{29-33}$ The Early Manifest Glaucoma Trial (EMGT) showed specifically for every $1 \mathrm{~mm} \mathrm{Hg}$ increase in pressure there was a $12-13 \%$ increased likelihood of

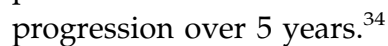

However, in exfoliation, pressures are even higher and progression more frequent even at normalized pressures. ${ }^{33}$ Just recently, further data from the EMGT showed that pressures in exfoliation tend to rise over time ( $1 \mathrm{~mm} \mathrm{Hg}$ per year) as opposed to primary openangle glaucoma, which remained stable. ${ }^{35}$ Consequently, in exfoliation a $1 \mathrm{~mm} \mathrm{Hg}$ decrease in pressure may be even more important than in primary open-angle glaucoma. However, prospective long-term studies are needed to confirm this speculation.
This study suggests that evening-dosed TTFC may provide greater mean 24-h IOP reduction, primarily at the 1800 hours time point, than the LTFC in XFG.

This study did not evaluate the long-term 24-h efficacy of the TTFC compared with LTFC in primary open-angle glaucoma or for the long-term visual outcomes between the two preparations. In addition, we did not evaluate directly morning dosing of these two fixed combinations. Further research is needed to more completely elucidate the efficacy of the TTFC and LTFCs compared with other fixed and unfixed therapies. This study was also limited by the crossover design which has threatens a carryover effect of the medicine between treatment periods. Although this effect was not observed statistically an influence from the first period medicines can never be completely rule-out. In addition because of the small 
samples size, an evaluation of safety could not be made as extensive as in a parallel design.

\section{Summary}

\section{What was known before}

- Published evidence suggests that because of the higher 24-h pressure curve characteristics associated with exfoliative glaucoma, further evaluating these patients might help elucidate if a difference exists in ocular hypotensive efficacy between two glaucoma preparations.

What this study adds

- This study suggests that evening dosed travoprost/ timolol fixed combination may provide greater mean $24-h$ intraocular pressure reduction, primarily at the 1800 hours time point, than the latanoprost/timolol fixed combination in exfoliative glaucoma.

\section{Conflict of interest}

Dr Stewart receives research funding from Alcon and has been a consultant, but does not receive honoraria or perform lectures, and received no support for this particular study. Professor Konstas receives research funding and lectures for Alcon. Drs Mikropoulos, Embeslidis, Dimopoulos, and Papanastasiou as well as Professor Haidich do not have conflict of interest to disclose.

\section{Acknowledgements}

The clinical/data collection site (AGP, Greece) and the administrative site (WCS, PRN Pharmaceutical Research Network, LLC) were solely responsible for design and development of the protocol as well as analysis and interpretation of data. Alcon was not involved in the protocol design, development, analysis, or editing process. The clinical/data collection site approached Alcon Laboratories, Inc. after the protocol was developed and received an unrestricted grant. The administrative site did not request or receive financial support for this study.

\section{References}

1 Barnebey HS, Orengo-Nania S, Flowers BE, Samples J, Mallick S, Landry TA et al. The safety and efficacy of latanoprost/timolol fixed combination $0.004 \%$ / timolol $0.5 \%$ fixed combination ophthalmic solution. Am J Ophthalmol 2005; 140: 1-7.

2 Pfeiffer N. A comparison of the fixed combination of latanoprost and timolol with its individual components. Graefes Arch Clin Exp Ophthalmol 2002; 240: 893-899.

3 Higginbotham EJ, Feldman R, Stiles M, Dubiner H. Latanoprost and timolol combination therapy vs monotherapy: one-year randomized trial. Arch Ophthalmol 2002; 120: 915-922.

4 Topouzis F, Melamed S, Danesh-Meyer H, Wells AP, Kozobolis V, Wieland $\mathrm{H}$ et al. A 1-year study to compare the efficacy and safety of once-daily travoprost $0.004 \%$ / timolol $0.5 \%$ to once-daily latanoprost $0.005 \%$ / timolol $0.5 \%$ in patients with open-angle glaucoma or ocular hypertension. Eur J Ophthalmol 2007; 17: 183-190.

5 Konstas AG, Tsironi S, Vakalis AN, Nasr MB, Stewart JA, Nelson LA et al Intraocular pressure control over 24-h using travoprost and timolol fixed combination, administered in the morning or evening in primary open-angle and exfoliative glaucoma. Acta Ophthalmol 2009; 87: 71-76.

6 Konstas AG, Boboridis K, Tzetzi D, Kallinderis K, Jenkins JN, Stewart WC. Twenty-four-hour control with latanoprost-timolol-fixed combination therapy $v \mathrm{~s}$ latanoprost therapy. Arch Ophthalmol 2005; 123: 898-902.

7 Konstas AG, Mikropoulos D, Haidich AB, Ntampos KS, Stewart WC. Twenty-four-hour intraocular pressure control with the travoprost/timolol maleate fixed combination compared with travoprost when both are dosed in the evening in primary open-angle glaucoma. $\mathrm{Br} J$ Ophthalmol 2009; 93: 481-485.

8 Konstas AG, Mikropoulos D, Stewart WC. Fixed combination therapy in glaucoma. In: Shaarawy TM, Sherwood MB, Hitchings RA, Crowston JG (eds). Glaucoma. Medical Diagnosis and Therapy. WB Saunders: Philadelphia, 2009; 565-575.

9 Konstas AG, Mantziris DA, Stewart WC. Diurnal intraocular pressure in untreated exfoliation and primary open-angle glaucoma. Arch Ophthalmol 1997; 115: 182-185.

10 Konstas AG, Mantziris DA, Cate EA, Stewart WC. Effect of timolol on the diurnal intraocular pressure in exfoliation and primary open-angle glaucoma. Arch Ophthalmol 1997; 115: 975-979.

11 Stewart WC, Holmes KT, Johnson MA. Washout periods for brimonidine $0.2 \%$ and latanoprost $0.005 \%$. Am J Ophthalmol 2001; 131: 798-799.

12 Book SA. Essentials of Statistics. McGraw Hill: New York, 1987; pp 205-215.

13 Simes RJ. An improved Bonferroni procedure for multiple tests of significance. Biometrika 1986; 73: 751-754.

14 Dupont WD, Plummer WD. PS power and sample size program available for free on the Internet. Controlled Clin Trials 1997; 18: 274.

15 Duff GR. A double-masked crossover study comparing the effects of carteolol 1 and $2 \%$ on intraocular pressure. Acta Ophthalmologica 1987; 65: 618-621.

16 Miller RG. Beyond ANOVA: Basics of Applied Statistics. Chapman \& Hall/CRC: London, 1998; pp 74-76.

17 Siegel S. Nonparametric Statistics for the Behavioral Sciences. McGraw Hill: New York, 1956; pp 63-67.

18 Konstas AG, Tsironi S, Ritch R. Current concepts in the pathogenesis and management of exfoliation syndrome and exfoliative glaucoma. Compr Ophthalmol Update 2006; 7: 131-141.

19 Konstas AG, Tsironi S, Kozobolis VP. Medical therapy of exfoliative glaucoma. In: Hollo G, Konstas AG (eds). "From Exfoliation Syndrome to Exfoliative Glaucoma" EGS Book. Dogma: Savona, 2008; pp 135-146.

20 Ritch R, Konstas AG, Schloetzer-Schrehardt U. Exfoliation syndrome and exfoliative glaucoma. In: Shaarawy TM, Sherwood MB, Hitchings RA, Crowston JG (eds). Glaucoma. 
Medical Diagnosis and Therapy. WB Saunders: Philadelphia, 2009; pp 339-347.

21 Konstas AG, Hollo G, Ritch R. Exfoliative glaucoma. In: Schacknow PN, Samples JR (eds). The Glaucoma Book. A Practical, Evidence-Based Approach to Patient Care. Springer: New York, 2010.

22 Konstas AG, Kozobolis VP, Katsimpris IE, Boboridis K, Koukoula S, Jenkins JN et al. Efficacy and safety of latanoprost versus travoprost in exfoliative glaucoma patients. Ophthalmology 2007; 114: 653-657.

23 Parmaksiz S, Yüksel N, Karabas VL, Ozkan B, Demirci G, Caglar Y. A comparison of travoprost, latanoprost, and the fixed combination of dorzolamide and timolol in patients with pseudoexfoliation glaucoma. Eur J Ophthalmol 2006; 16 : 73-80.

24 Konstas AG, Hollo G, Tsironi S, Durukan I, Goldenfeld M, Melamed S. Diurnal intraocular pressure control with bimatoprost vs latanoprost in exfoliative glaucoma: a crossover observer-masked 3-center study. $\mathrm{Br} J$ Ophthalmol 2007; 91: 757-760.

25 Konstas AG, Maltezos AC, Gandi S, Hudgins AC, Stewart WC. Comparison of 24-h intraocular pressure reduction with two dosing regimens of latanoprost and timolol maleate in patients with primary open-angle glaucoma. Am J Ophthalmol 1999; 128: 15-20.

26 Konstas AG, Nakos E, Tersis I, Lallos NA, Leech JN, Stewart WC. A comparison of once-daily morning vs evening dosing of concomitant latanoprost/timolol. Am J Ophthalmol 2002; 133: 753-757.

27 Tabet R, Stewart WC, Feldman R, Konstas AG. A review of additivity to prostaglandin analogs: fixed and unfixed combinations. Surv Ophthalmol 2008; 53: S85-S92.
28 Sharif NA, Kelly CR, Crider JY, Williams GW, Xu SX. Ocular hypotensive FP prostaglandin (PG) analogs: PG receptor subtype binding affinities and selectivities, and agonist potencies at FP and other PG receptors in cultured cells. J Ocul Pharmacol Ther 2003; 19: 501-515.

29 Stewart WC, Konstas AG, Nelson LA, Kruft B. Metaanalysis of 24-h intraocular pressure studies evaluating the efficacy of glaucoma medicines. Ophthalmology 2008; 115: 1117-1122.

30 Stewart WC, Kolker AE, Sharpe ED, Day DG, Holmes KT, Leech JN et al. Factors associated with long-term progression or stability in primary open-angle glaucoma. Am J Ophthalmol 2000; 130: 274-279.

31 Stewart WC, Chorak RP, Hunt HH, Sethuraman G. Factors associated with visual loss in patients with advanced glaucomatous changes in the optic nerve head. Am J Ophthalmol 1993; 116: 176-181.

32 Mao LK, Stewart WC, Shields MB. Correlation between intra-ocular pressure control and progressive glaucomatous damage in primary open-angle glaucoma. Am J Ophthalmol 1991; 111: 51-55.

33 Konstas AG, Hollo G, Astakhov YS, Teus MA, Akopov EL, Leech JN, Stewart WC. Factors associated with long-term progression or stability in exfoliation glaucoma. Arch Ophthalmol 2004; 122: 29-33.

34 Leske MC, Heijl A, Hyman L, Bengtsson B, Dong L, Yang Z et al EMGT Group. Predictors of long-term progression in the early manifest glaucoma trial. Ophthalmology 2007; 114: 1965-1972.

35 Hyman L, Heijl A, Leske MC, Bengtsson B, Yang Z, Early Manifest Glaucoma Trial Group. Natural history of intraocular pressure in the early manifest glaucoma trial: a 6-year follow-up. Arch Ophthalmol 2010; 128: 601-607. 